University of Nebraska - Lincoln

DigitalCommons@University of Nebraska - Lincoln

Faculty Publications in Computer \& Electronics Electrical \& Computer Engineering, Department Engineering (to 2015)

2011

\title{
Power Controlled Coded-Sequence Self-Encoded Spread Spectrum Communications
}

\author{
Poomathi Duraisamy \\ University of Nebraska-Lincoln \\ Liang Chi \\ University of Nebraska at Omaha, Ichi@unomaha.edu \\ Won Mee Jang \\ University of Nebraska-Lincoln, wjang1@unl.edu \\ Lim Nguyen \\ University of Nebraska-Lincoln, Inguyen1@unl.edu
}

Follow this and additional works at: https://digitalcommons.unl.edu/computerelectronicfacpub

Part of the Computer Engineering Commons

Duraisamy, Poomathi; Chi, Liang; Jang, Won Mee; and Nguyen, Lim, "Power Controlled Coded-Sequence Self-Encoded Spread Spectrum Communications" (2011). Faculty Publications in Computer \& Electronics Engineering (to 2015). 99.

https://digitalcommons.unl.edu/computerelectronicfacpub/99

This Article is brought to you for free and open access by the Electrical \& Computer Engineering, Department of at DigitalCommons@University of Nebraska - Lincoln. It has been accepted for inclusion in Faculty Publications in Computer \& Electronics Engineering (to 2015) by an authorized administrator of DigitalCommons@University of Nebraska - Lincoln. 


\title{
Power Controlled Coded-Sequence Self-Encoded Spread Spectrum Communications
}

\author{
Poomathi Duraisamy, Liang Chi, Won Mee Jang, Lim Nguyen \\ Department of Computer and Electronics Engineering \\ The Peter Kiewit Institute of Information Science, Technology and Engineering \\ University of Nebraska-Lincoln \\ Omaha, NE - 68182
}

\begin{abstract}
In this paper, we propose to apply power control to coded-sequence self-encoded spread spectrum (CS-SESS) communications in dynamic fading channels. We determine the statistical characteristics of the power enhancement in the transmitter due to power control and show that the proposed approach improves the power efficiency in mobile cellular systems.

In addition, we propose to mitigate self interference in CSSESS by exploiting the information that the transmitter obtains, through feedback training sequences, about the de-spreading code in the receiver. The results show that the mitigation is significant even with only selective training chips from the de-spreading sequence. Similar improvement can be achieved with feed-forward training sequences to the receiver about the spreading code in the transmitter.
\end{abstract}

Index Terms - spread spectrum, power control, iterative detection, coded-sequence self-encoded spread spectrum.

\section{INTRODUCTION}

Coded-sequence self-encoded spread spectrum derives its spreading codes from the random information source rather than using traditional pseudo-random codes. In recent work, we have shown that with iterative detection, the memory in CS-SESS modulated signals not only can deliver a $4.7 \mathrm{~dB}$ gain in AWGN channels, but also can be exploited to achieve time diversity and robust bit-error rate (BER) performance in fading channels [3]. In this paper, transmitter precoding, as an alternative to combat the channel dynamic of CS-SESS communications in dynamic fading channel is proposed and analyzed. Transmitter precoding was originally proposed for additive white Gaussian noise (AWGN) channels [4, 5] and independently for flat fading channels in [6].

Although, CDMA based systems provide high power efficiency and moderate error rates, coherent modulation does not provide reliable communication on fading channels if the impulse responses of these channels are not known. Traditionally, channel estimation can be achieved by periodical transmission of known training sequences or using pilot channel. Generally, for better estimation accuracy, more training symbols or higher power for pilot channel shall be required [1]. In this paper, we exploit the training sequence in CS-SESS communication to combat the self interference that attenuate the strength of the de-spread signal at low SNR. We study three scenarios

1) Transmitter exploits the de-spreading code of the receiver obtained through feedback training sequence

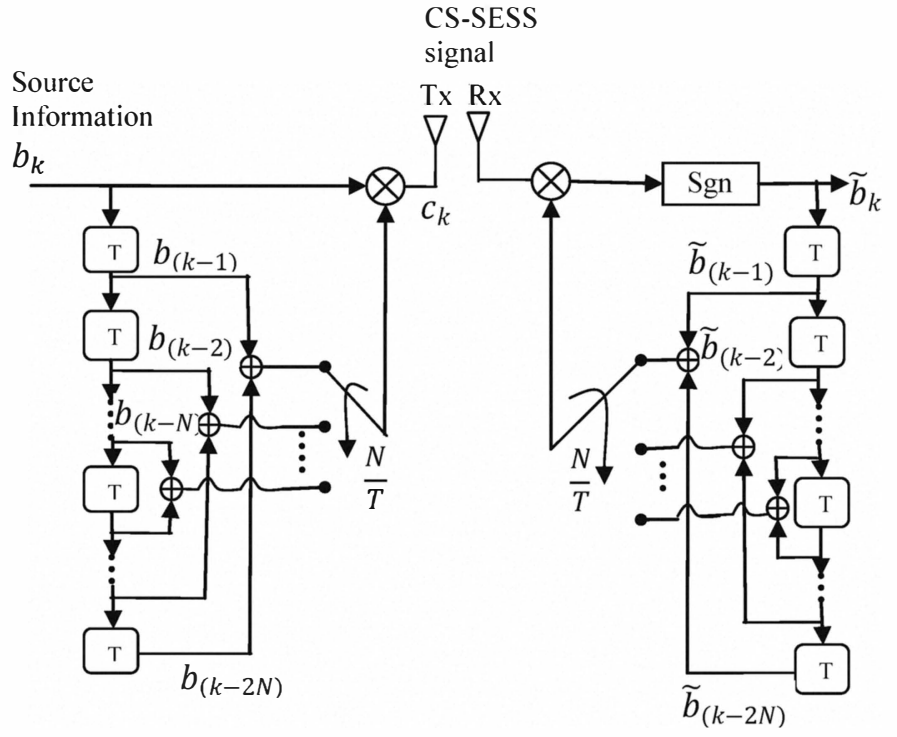

Fig. 1. Block diagram of coded-sequence self-encoded spread spectrum

2) Receiver exploits the spreading code of the transmitter obtained through feedforward training sequence

3) Receiver corrects the most significant de-spreading sequence errors by exploiting both feed forward and feedback training sequence.

We showed that the mitigation of self interference is significant in all three cases.

The rest of the paper is organized as follows. In section II, we describe the CS-SESS scheme with dynamic fading channel. Power control to combat channel dynamic is explained in section III with the BER performance results. In section IV, we explain three scenarios that exploit the training sequence to mitigate self interference and showed its numerical results followed by conclusion in section $\mathrm{V}$.

\section{SYSTEM MODEL}

\section{A. Transmitter}

Fig. 1 illustrates the schematic block diagram of the proposed CS-SESS. $T$ is the bit duration and $T_{c}$ is the chip duration. The source information $b$ is assumed to be bipolar values. These symbols are stored sequentially in $2 N$ delay 
shift registers. The delayed symbols are then encoded by means of modulo- 2 adders to generate the spreading sequence $s_{k}$ of length $N$ that modulate the current symbol at the chip rate $N / T$. For example, the spreading sequence for the $k^{t h}$ bit $b_{k}$ is given as

$$
s_{k}=\left[\begin{array}{c}
b_{(k-1)} \oplus b_{(k-2 N)} \\
b_{(k-2)} \oplus b_{(k-2 N+1)} \\
\vdots \\
b_{(k-N)} \oplus b_{(k-N-1)}
\end{array}\right]
$$

Thus, with a random input data stream, the sequence is also random and time-varying from one bit to another. The spread chips of bit $b_{k}$ are then transmitted as

$$
c_{k}=b_{k} s_{k}
$$

If $c_{k n}$ is the $n^{\text {th }}$ chip of spread sequence $c_{k}$ and $s_{k n}$ is the $n^{t h}$ chip of spreading sequence $s_{k}$ then

$$
c_{k n}=b_{k} s_{k n}=b_{k} b_{(k-n)} b_{(k-2 N-1+n)}
$$

Note that the XOR operator is omitted in (3) because of its similiarity to multiplication in bipolar case.

\section{B. Dynamic Fading Channel}

The Rayleigh fading can be expressed using two random variables $a$ and $b$ as

$$
h=\frac{a^{2}+b^{2}}{\sqrt{2}}
$$

We assume the channel is dynamic by the variance $X$ which varies from 0 to 2 . Dynamic Rayleigh fading can be expressed as

$$
h=\sqrt{X}\left[\frac{a^{2}+b^{2}}{\sqrt{2}}\right]
$$

\section{Correlation Detection}

The receiver performs the reverse operation for symbol recovery by means of a correlation detector. If $\tilde{c}_{k n}$ is the received signal

$$
\tilde{c}_{k n}=h_{k} c_{k n}+\zeta_{k n}
$$

where the additive Gaussian noise $\zeta_{k n}$ has the zero mean and variance $N N_{0} / 2$. Note that the variance is narrow-band noise variance $N_{0} / 2$ multiplied by the spreading factor $N$, then the correlation estimate $\tilde{b}_{k}$ of bit $b_{k}$ is given as

$$
\tilde{b}_{k}=\tilde{c}_{k} \tilde{s}_{k}=\sum_{n=1}^{N} \tilde{c}_{k n} \tilde{s}_{k n}
$$

where $\tilde{s}_{k n}$ is the $n^{t h}$ de-spreading chip for $k^{t h}$ bit generated by appropriately modulo- 2 adding the delay registers. We assume that the content of the delay registers in transmitter and receiver should be identical at the start of the data transmission. Notice that the initial synchronization and a complete knowledge of the delay-register structure could make data recovery difficult for an unintended receiver. By substituting (6) and (3) into the correlation estimate given in (7), we have

$$
\begin{gathered}
\tilde{b}_{k}=h_{k}\left[\sum_{n=1}^{N} b_{(k-n)} b_{(k-2 N-1+n)} \tilde{b}_{(k-n)} \tilde{b}_{(k-2 N-1+n)}\right] b_{k} \\
+\sum_{n=1}^{N} \zeta_{k n} \tilde{s}_{k n}
\end{gathered}
$$

The content of delay registers are then updated by the hard decision of the correlation estimate $\tilde{b}_{k}$.

$$
\tilde{b}_{k}=\operatorname{sgn}\left(\tilde{b}_{k}\right)= \begin{cases}1, & \text { if } \tilde{b}_{k}>0 \\ -1, & \text { if } \tilde{b}_{k}<0\end{cases}
$$

Since there may be errors in correlation estimate, the reconstructed de-spreading sequence could be different from the spreading sequence at the transmitter. A bit error therefore will propagate through the delay registers for the next $2 N$ bits instead of $N$ bits in SESS and cause self interference (expressed by the term inside the square bracket in (8)) that attenuates the strength of the despread signal.

\section{Iterative Detection}

The iterative detector, like the MLSE detector, also improves the system performance by exploiting a sequence of received signals. However, like the feedback detector, the complexity of the iterative detector is linear with $N$.

We begin by expressing the spread sequence $c_{k}$ of $2 N+1$ bits $b_{1}, b_{2} \ldots b_{(2 N+1)}$ in a matrix as shown in (10). Based on (3) the spread chips can be written in $2 N+1 \times N$ block, since $N$ is the spreading length. Thus, each element of right hand side matrix of equation (10) represent a chip and $j^{\text {th }}$ row includes the chips of bit $b_{j}$ (also can be expressed as chips $c_{j 1}, c_{j 2} \ldots$ $\left.c_{j N}\right)$. In the transmitter, row by row each spreading sequence is transmitted.

In the receiver, $\tilde{c}_{k}$ of $c_{k}$ is received. We begin by decoding the first bit $b_{1}$. Observe from the transmitted chips of (10), the current decoded symbol $b_{1}$ not only depends upon the previous $2 N$ symbols $\left(b_{0}, b_{-1} \cdots b_{(1-2 N)}\right)$ as indicated in the first row of (10), which can be decoded using correlation detection. But $b_{1}$ also can be re-estimated from future $2 N$ chips $\left(c_{21}, c_{32} \cdots c_{(N+1) N}, c_{(N+2) N} \cdots c_{(2 N+1) 1}\right)$ of (10) from $2 N$ future transmitted symbols $\left(c_{2}, c_{3} \cdots c_{(2 N+1)}\right)$ respectively. This re-estimation of bit from $2 N$ future symbols can be termed as time diversity detection. This is due to the fact that bit $b_{k}$ stays in the delay register for next $2 N$ bits $\left(b_{(k+1)}, b_{(k+2)} \ldots . . b_{(k+2 N)}\right)$ spreading. Also note from (10), the time diversity estimate of $b_{1}$ requires previous iterated bits $\left(b_{(-2 N+2)}, b_{(-2 N+4)} \cdots b_{0}\right)$ and also future correlation detected bits $\left(b_{2}, b_{3} \cdots b_{(2 N+1)}\right)$.

Thus, the time diversity estimate of $b_{1}$ from $2 N$ future symbols can be used to improve the estimate of the current symbol $b_{1}$ from the correlation detection. If $\tilde{c}_{k n}$ is the estimated value of $c_{k n}$ at the receiver, the iterative detection soft estimate $\tilde{\tilde{b}}_{k}$ can be obtained by adding the correlation and time diversity estimates as shown in (11). The output of the first iteration is 


$$
\begin{aligned}
& {\left[\begin{array}{c}
c_{1} \\
c_{2} \\
\vdots \\
c_{(N+1)} \\
c_{(N+2)} \\
\vdots \\
c_{(2 N+1)}
\end{array}\right]=\left[\begin{array}{cccc}
b_{1} b_{0} b_{(1-2 N)} & b_{1} b_{-1} b_{(-2 N+2)} & \cdots & b_{1} b_{(1-N)} b_{-N} \\
b_{2} b_{1} b_{(-2 N+2)} & b_{2} b_{0} b_{(-2 N+3)} & \cdots & b_{2} b_{(2-N)} b_{(-N+1)} \\
\vdots & \vdots & \ddots & \vdots \\
b_{(N+1)} b_{N} b_{(1-N)} & b_{(N+1)} b_{(N-1)} b_{(-N+2)} & \cdots & b_{(N+1)} b_{1} b_{0} \\
b_{(N+2)} b_{(N+1)} b_{(2-N)} & b_{(N+2)} b_{N} b_{(-N+3)} & \cdots & b_{(N+2)} b_{2} b_{1} \\
\vdots & \vdots & \ddots & \vdots \\
b_{(2 N+1)} b_{(2 N)} b_{1} & b_{(2 N+1)} b_{(2 N-1)} b_{2} & \cdots & b_{(2 N+1)} b_{(N+1)} b_{N}
\end{array}\right]}
\end{aligned}
$$

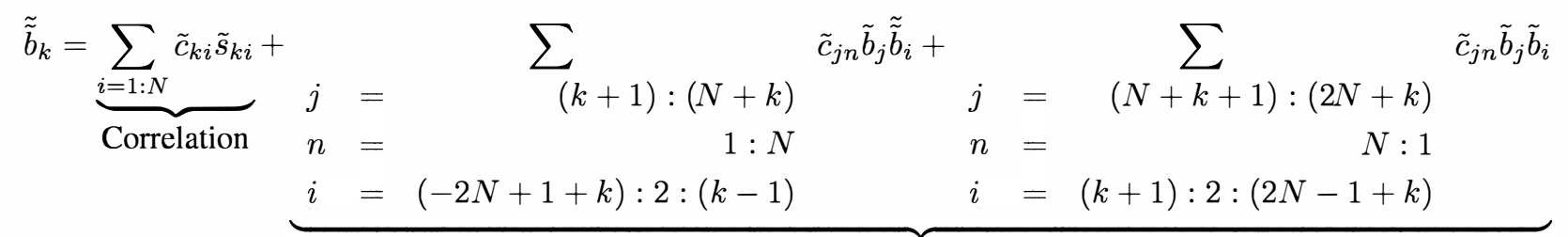

Time diversity output

then obatined by the hard decision of $\tilde{\tilde{b}}_{k}$. It is clear that the number of chips employed by the above iteration in making a bit decision is $3 N$ chips instead of $2 N$ chips in SESS. As a result, an improvement of system performance can be reasonably expected. Since $2 N$ future symbol decisions are needed for an iteration of the current symbol, there will be a delay of $2 N$ symbols for each iteration.

\section{CS-SESS POWER CONTROL}

\section{A. Power Control}

Precoding is done to combat the channel dynamics. The crucial assumption for precoding is that the transmitter has information about the channel between it and the active receiver. This information can be obtained from the receiver via feedback channel or can be estimated at the transmitter when a time-division duplex mode is employed, that is, when the same frequency band is employed for transmit and receive direction. Another important requirement is that channel is sufficiently slow that it remains essentially constant over the bit duration. We apply the power control factor $(1 / \sqrt{X})$ at the transmitter as

$$
c_{k}=\frac{1}{\sqrt{N}} b_{k} s_{k}
$$

The Fig. 2 shows the histogram of the power control variable. It is clear from the figure that most of the values are concentrated between 0.5 and 10 .

The mean and variance of power control variable can be found as

$$
\begin{gathered}
E\left[\frac{1}{N}\right]=3.03=4.8 \mathrm{~dB} \\
\operatorname{Var}\left[\frac{1}{X}\right]=E\left[\frac{1}{X^{2}}\right]-E\left[\frac{1}{X}\right]^{2}=163.885=22 \mathrm{~dB}
\end{gathered}
$$

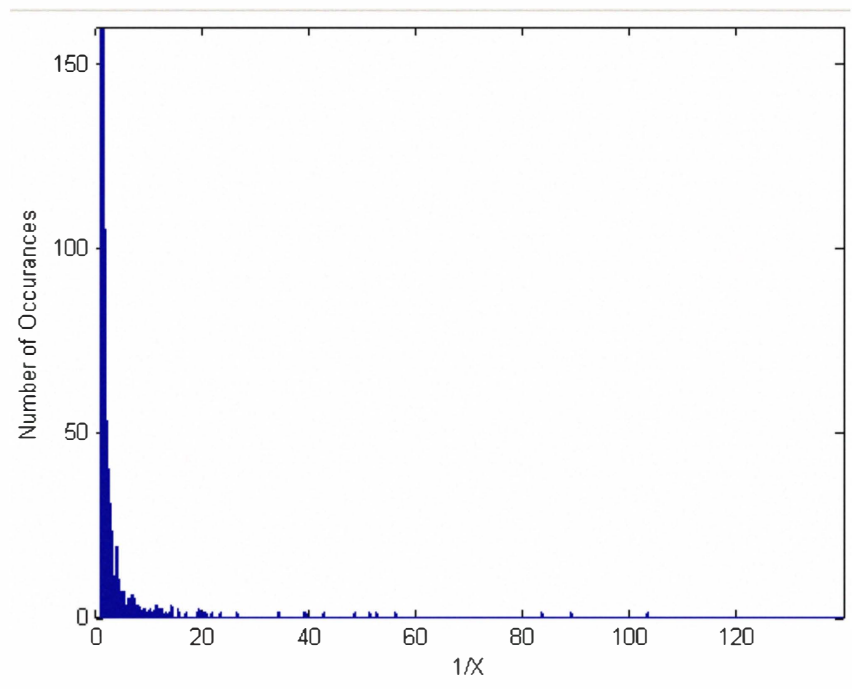

Fig. 2. Histogram of power control variable

\section{B. Results}

The simulations were done for $N=64$ unless otherwise specified. Fig. 3 shows the performance of correlation detector of Fig. 1 under dynamic fading channel with and without power control. The plot shows that the performance gain of $\sim 5 \mathrm{~dB}$ at $10^{-2}$ BER has been achieved with power control. As we discussed, the degradation at lower SNR is caused by error propagation, such that the bit errors effectively contribute to an additional attenuation of the de-spread signal. Also note that the performance becomes similar to that of BPSK of Rayleigh fading channel for SNR $>10 \mathrm{~dB}$ by fully eliminating the channel dynamics.

Fig. 4 shows the BER performance of iterative detector with and without power control in dynamic fading channel. It can 


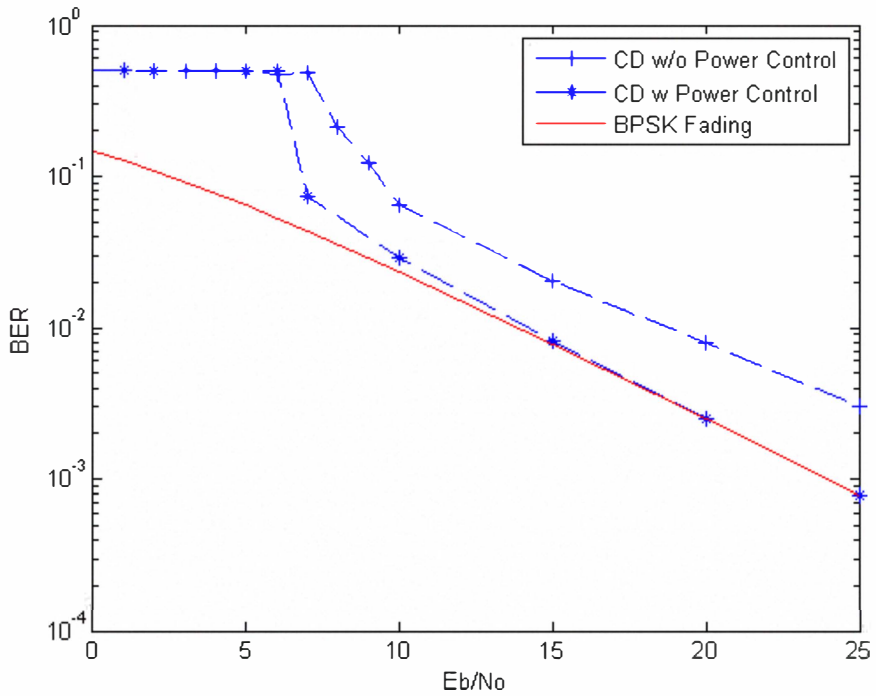

Fig. 3. BER performance of correlation detector in dynamic fading channel

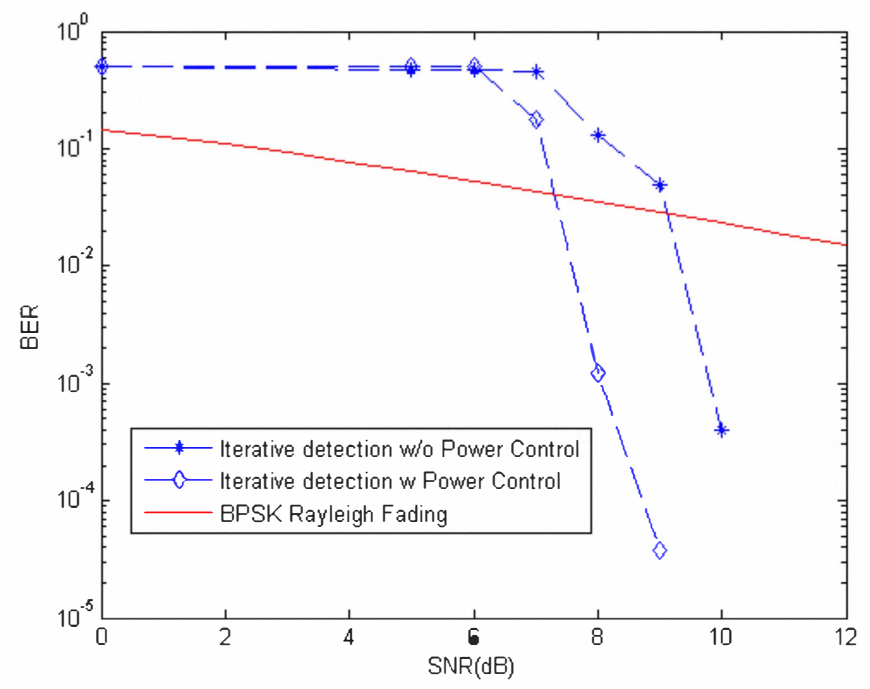

Fig. 4. BER performance of iterative detection in dynamic fading channel

be seen that power control can extract additional gain of about $1.5 \mathrm{~dB}$ at $10^{-2}$ BER. Performance degradation at lower SNR is because of its nature to use correlation detector output to re-estimate the current bit. A comparison of the plots in Figs. 3 and 4 further demonstrates that iterative detector can attain performance gain of $\sim 17 \mathrm{~dB}$ at $10^{-3}$ BER with power control.

\section{CS-SESS with TRaining SEquence}

Training sequence is often used in wireless communication systems to estimate the slowly time-varying channel. Here we propose three scenarios to mitigate the self interference in CSSESS by exploiting the

1) Feedback training sequence to convey de-spreading code of the receiver to the transmitter

2) Feed-forward training sequence to convey spreading sequence of the transmitter to receiver

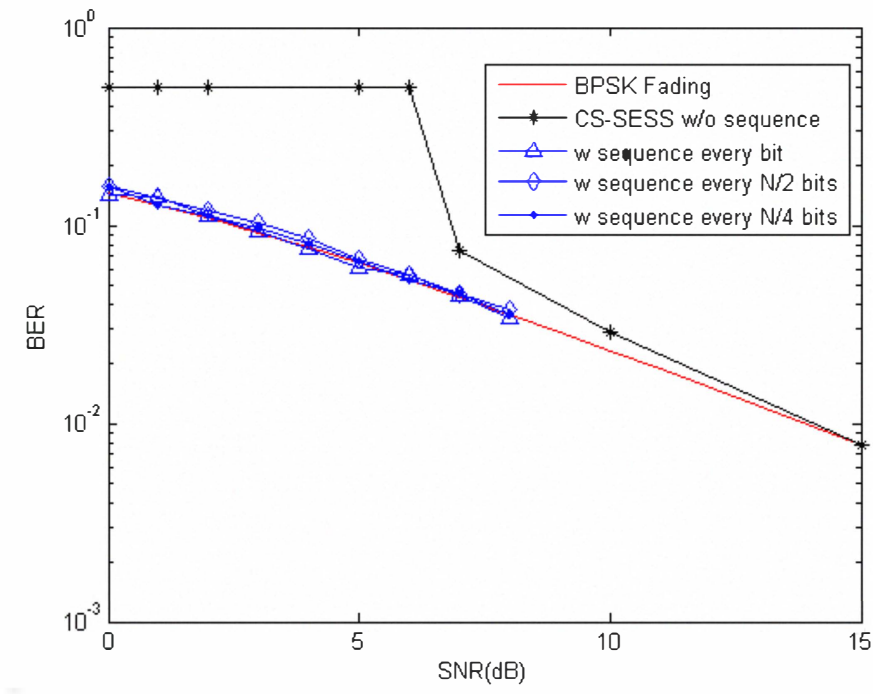

Fig. 5. BER performance of correlation detection using feedback training sequence

3) Both feedback and feed-forward training sequence to communicate between transmitter and receiver

We assume that these high power training sequence are error free.

\section{A. Feedback Training Sequence}

We assume that the transmitter's delay registers are updated by the de-spreading sequence of the receiver, obtained through the feedback training sequence. Fig. 5 shows the performance of the correlation detection with and without using the feedback training sequence in Rayleigh fading channel. Simulations results were obtained for exploiting feedback training sequence once every bit, $N / 2$ or $N / 4$ bit transmissions. The plot shows that the self interference can be eliminated completely at lower SNR. Also note that the performance of receiving feedback training sequence, every $N / 2$ or $N / 4$ bit transmissions is also almost similar to BPSK due to the fact that updated de-spreading code in the delay registers of the transmitter stays until it is flushed out.

Fig. 6 shows the performance of iterative detection with and without using the feedback training sequence. The result shows that the performance is same as the correlation detector with no additional time diversity gain of iterative detection. This is due to the loss of original source information in the transmitter delay register to spread the future $2 N$ bits which can be used later in the receiver to re-estimate the current bit using time diversity detection.

1) Modified Method For Feedback Training Sequence: Above method of using the de-spreading sequence helps the correlation detection but it eliminates the use of iterative detection. So we proposed an modified method with below algorithm

1) Get the de-spreading sequence from the receiver once every bit

2) Compare with spreading sequence 


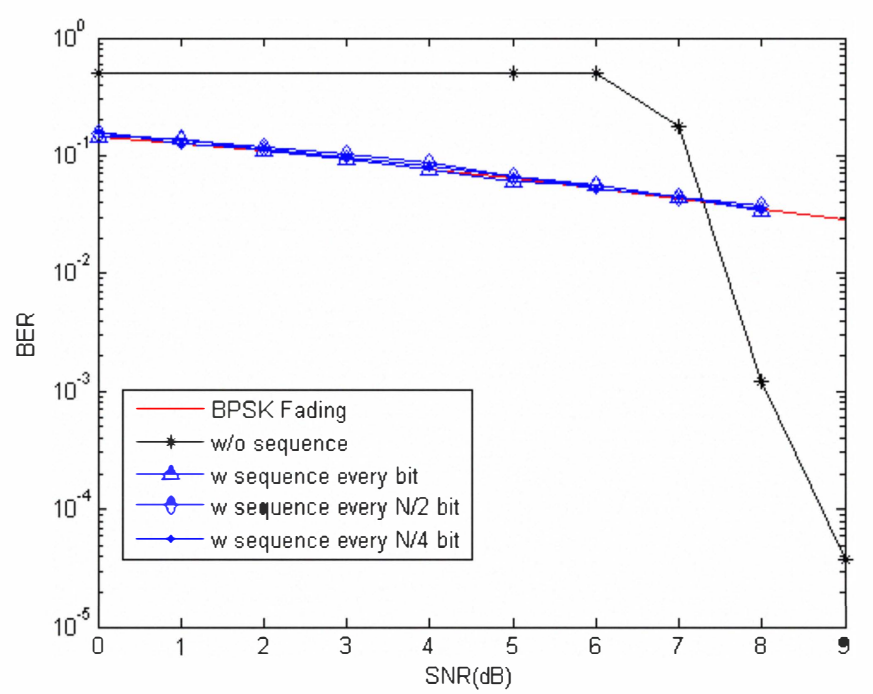

Fig. 6. BER performance of iterative detection using feedback training sequence

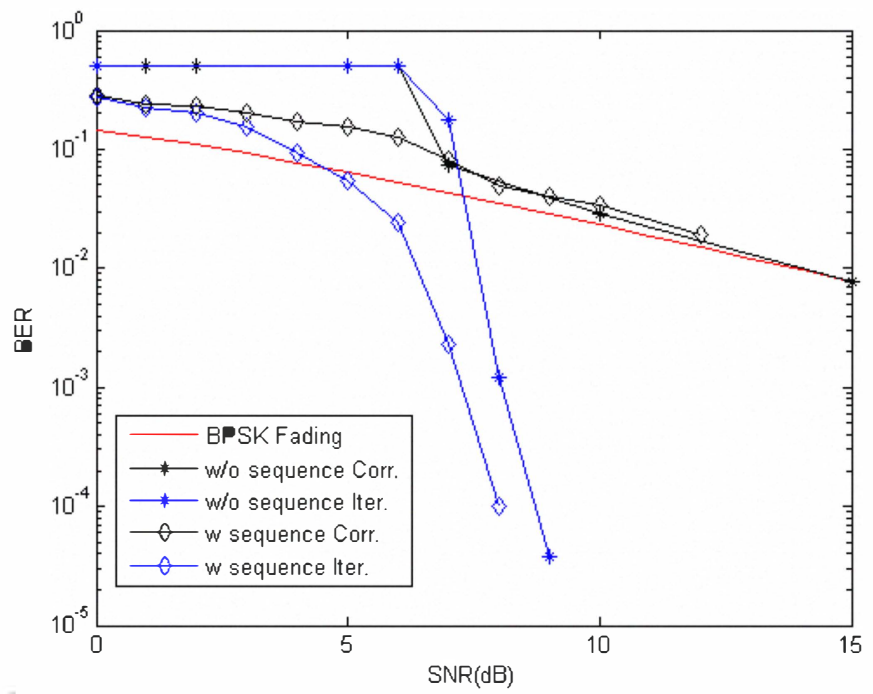

Fig. 7. BER performance of iterative detection using feedback training sequence

3) If Number of disagreed chips $\geq N / 4$

a) Then flip the sign of the each spread sequence chips until number of disagreed chips is $\leq N / 4$

b) Don't change the values of the delay registers of the transmitter

4) Else follow the regular the spreading process

This method will not change every error chips to coincide with de-spreading sequence instead it leaves $N / 4$ of the wrong chips intact, which in-turn helps iterative detection, by preventing the lose of $N / 4$ original source chips.

CS-SESS Performance using the modified method is shown in Fig. 7. Although, correlation detection performance is not similar to BPSK fading, this method clearly helps for iterative detection. It is clear that iterative detection is able to attain gain of $\sim 1 \mathrm{~dB}$ at $10^{-4}$ BER beyond eliminating the self-interference

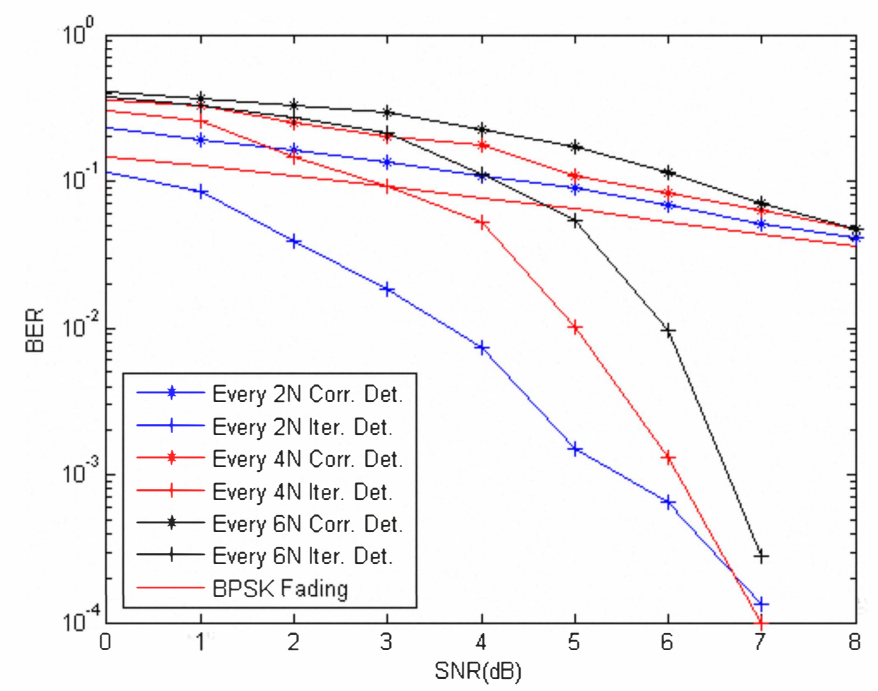

Fig. 8. BER performance using feed-forward training sequence

in low SNR.

\section{B. Feed-forward Training Sequence}

We assume that the receiver can obtain the spreading sequence of the transmitter through feed-forward training sequence. Fig. 8 shows the BER performance with and without utilizing the feed-forward training sequence to update the receiver with the spreading sequence for every $2 N, 4 N$ or $6 N$ bit transmissions, which is $50 \%, 66 \%$ or $75 \%$ throughput respectively. It is clear that using feed-forward training sequence eliminates the self-interference depending upon the frequency of the update. Note that the update every $2 N$ bit transmissions can eliminate the self-interference completely. A comparison of Figs. 7 and 8 further demonstrate that iterative detection using feed-forward training sequence with less frequent update of every $6 N$ bit transmission not only have higher bandwidth efficiency but also provides significant performance improvement.

\section{Feedback and Feed-forward Training Sequence}

Sending the whole spreading or de-spreading sequence often in training sequence is not practically possible, so we tried to send the location of the 4,6 or 8 most significant mismatched delay register bits in the de-spreading sequence for every $2 N$ bit transmission. We assume that transmitter obtains the de-spreading chips of the receiver through feedback training sequence to compare and send the delay register error locations. Receiving the error bit locations, the receiver can invert their values to match with spreading sequence.

Fig. 9 shows the performance of CS-SESS for 4, 6 and 8 error corrections in the receiver. Correcting 4 and 6 errors in the total of 64 delay registers does not show any performance improvement. Correcting 8 error in the de-spreading sequence shows a $1 \mathrm{~dB}$ improvement in self-interference. 


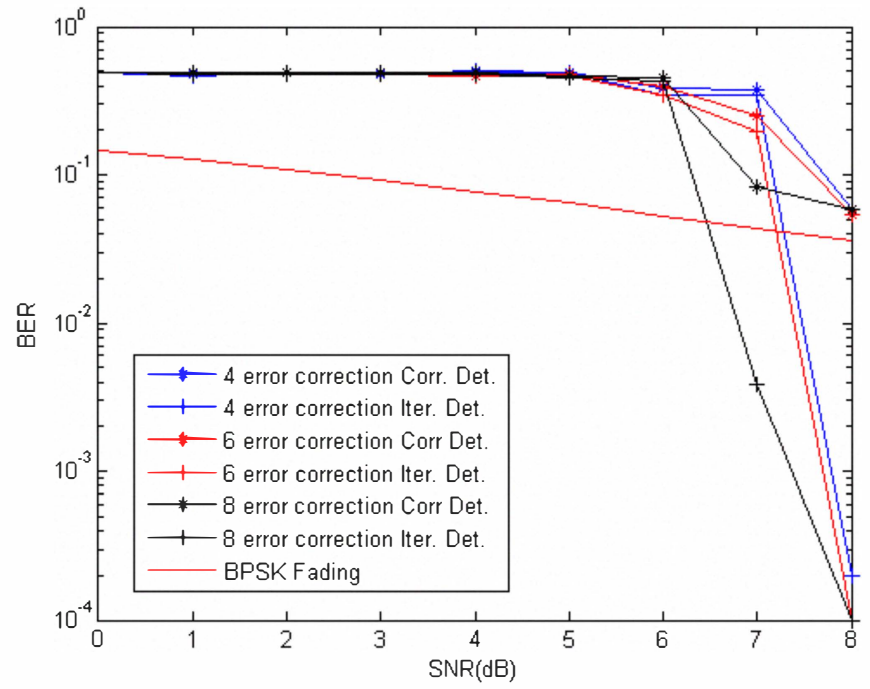

Fig. 9. BER performance using both feedback and feed-forward training sequence

\section{CONCLusion}

In this paper, we proposed the transmitter precoding to combat the channel dynamics of CS-SESS communication. Simulations showed that the performance gain of $5 \mathrm{~dB}$ and $1.5 \mathrm{~dB}$ for $\mathrm{SNR}>6 \mathrm{~dB}$ can be achieved with power control in correlation detection and iterative detection respectively in dynamic fading channel.

In addition, performance of CS-SESS was studied under three scenario of exploiting training sequence to mitigate the self interference in Rayleigh fading channel. The results showed that using feedback training sequence can eliminate the self interference in correlation detection. The proposed modified method to use feedback training sequence not only eliminates the self-interference in low SNR of correlation detection but helps iterative detection to attain additional $1 \mathrm{~dB}$ gain compared to original scheme. Exploiting feed-forward training sequence to obtain the spreading chips of the transmitter in the receiver can improve the performance significantly for less frequent training sequence by increasing the bandwidth efficiency.

\section{ACKNOWLEDGMENT}

This work was funded in part by contract award FA9550-081-0393 from the U.S. Air Force Office of Scientific Research. We wish to thank Dr. Jon Sjogren for his support of this study.

\section{REFERENCES}

[1] J. G. Proakis, Digital Communications, McGraw-Hill, New York, NY, 1983.

[2] L. Nguyen, "Self-encoded spread spectrum communications", Proc. IEEE MILCOM99, vol. 1, pp. 182-186, 1999.

[3] P. Duraisamy, L. Nguyen, "Coded-Sequence Self-encoded spread spectrum Communications", Proc. IEEE GLOBECOM 2009,pp 1-5, 2009.

[4] B. R. Vojcic, "Transmitter precoding in multiuser communications," Proc. 1995 IEEE IT Workshop on Information Theory, Multiple Access and Queueing Theory, St. Louis, MO, 1995.
[5] — " "Transmitter precoding in synchronous multiuser communications," in Symp. on Mobility Management, George Mason Univ., Fairfax, VA, 1994.

[6] Z. Tang and S. Cheng,"Interference cancellation for DS-CDMA systems over flat fading channels through pre-decorrelating," in PIMRC' 94, The Hague, The Netherlands, 1994.

[7] R. L. Peterson, R. E. Ziemer and David E. Borth, Introduction to Spread Spectrum Comminucations, Englewood Cliffs, NJ: Prentice Hall, 1995

[8] Y. Kong, L. Nguyen, and W. M. Jang, "Self-encoded spread spectrum modulation with differential encoding", Proc. IEEE 7-th ISSSTA 2002, vol. 2, pp. 471-474, 2002.

[9] Y. S. Kim, W. M. Jang, Y. Kong and L. Nguyen, "Chip-interleaved Self-encoded multiple access with iterative detection in fading channel", Journal of Communications and Networks, vol. 9, no. 1, pp. 50-55, Mar 2007 